\title{
Neurological outcome of patients with cryopyrin-associated periodic syndrome (CAPS)
}

\author{
Nafissa Mamoudjy ${ }^{1}$, Hélène Maurey ${ }^{3}$, Isabelle Marie ${ }^{2}$, Isabelle Koné-Paut ${ }^{2}$ and Kumaran Deiva ${ }^{3 *}$
}

\begin{abstract}
Background: To assess the neurological involvement and outcome, including school and professional performances, of adults and children with cryopyrin-associated periodic syndrome (CAPS).

Methods: In this observational study, patients with genetically proven CAPS and followed in the national referral centre for autoinflammatory diseases at Bicêtre hospital were assessed. Neurological manifestations, CSF data and MRI results at diagnosis and during follow-up were analyzed.

Results: Twenty-four patients ( 15 adults and 9 children at diagnosis) with CAPS were included. The median age at disease onset was 0 year (birth) [range 0-14], the median age at diagnosis was 20 years [range 0-53] and the mean duration of follow-up was $10.4 \pm 2$ years. Neurological involvement at diagnosis, mostly headaches and hearing loss, was noted in 17 patients (71\%). Two patients of the same family had abnormal brain MRI. A439V mutation is frequently associated with a non-neurological phenotype while R260W mutation tends to be associated with neurological involvement. Eleven adult patients (61\%) and 3 children (50\%) underwent school difficulties.

Conclusion: Neurological involvement is frequent in patients with CAPS and the majority of patients presented difficulties in school performances with consequences in the professional outcome during adulthood. Further studies in larger cohorts of children with CAPS focusing in intellectual efficiency and school performances are necessary.
\end{abstract}

Keywords: CAPS syndrome, Brain, Children, Outcome, Cognitive

\section{Background}

Cryopyrin-associated periodic syndrome (CAPS) is a rare hereditary periodic fever syndrome with an estimated prevalence in France equal to 1/360 000 [1]. CAPS are caused by dominantly inherited, or de novo, gain of function mutations within the NLRP3 gene [1,2]. NRLP3 encodes cryopyrin, which controls the activation of caspase-1 which in turn catalyses the cleavage of prointerleukin-1 $\beta$ (IL-1 $\beta$ ) into the potent proinflammatory cytokine IL-1 $\beta$ [3]. Mutations of NRLP3 are associated

\footnotetext{
*Correspondence: kumaran.deiva@aphp.fr

${ }^{3}$ Assistance Publique-Hôpitaux de Paris, Hôpitaux Universitaires Paris Sud, Pediatric Neurology Department and National Referral Center for Neuroinflammatory Diseases in Children and Inserm UMR 1184, Center for immunology of viral infections and autoimmune diseases, CEA, IDMIT, University Paris Sud, 63, rue Gabriel Péri, 94276 Le Kremlin-Bicêtre Cedex, France

Full list of author information is available at the end of the article
}

with overactivation of the inflammasome and thus overexpresion of IL-1 $\beta$. Because of this overproduction of IL-1, a specific treatment of CAPS using anti- IL-1 $\beta$ monoclonal antibodies such as canakinumab which is a human antiinterleukin-1 $\beta$ monoclonal antibody are used [4].

The syndrome encompasses a continuum of three diseases, from the mildest familial cold autoinflammatory syndrome (FCAS) to the most severe Neonatal Onset Multisystem Inflammatory Disease (NOMID) also known as Chronic Infantile Neurologic Cutaneous Articular (CINCA) syndrome. The Muckle-Wells syndrome (MWS) has an intermediate phenotype $[5,6]$. The clinical manifestations of CAPS such as urticaria-like skin rash, eyes redness, myalgia and sensory deafness, are not specific, if considered separately, and that often leads to a diagnostic delay which compromises the quality of life and exposes the patients to neurosensory complications 
and renal failure by secondary amyloidosis [5, 7, 8]. Central nervous system manifestations in this syndrome can also occur with various abnormalities on brain MRI [6]. Patients with leptomeningeal or dural enhancement had significantly lower IQ levels than did patients without enhancement. These manifestations are not well known especially by clinicians with a possible underestimation of neurological involvement [9].

The aim of our study is to assess neurological involvement and outcome, including school and professional performances, especially under canakinumab treatment of adults and children with CAPS.

\section{Methods}

\section{Patients}

We analyzed retrospectively data of patients with genetically proven CAPS, followed in the referral center for rare autoinflammatory diseases from January 2002 to January 2015. We identified 24 patients and collected demographic data, age at onset, age at diagnosis and clinical characteristics: fever, skin involvement, musculo-skeletal and ocular manifestations. All patients were followed since their childhood and underwent at least one clinical examination by a neurologist or a pediatric neurologist. Neurological manifestations were defined by any clinical symptoms of neurological involvement: headaches, meningeal syndrome, sensorineural deafness (confirmed by audiograms), optic nerve involvement (based on funduscopic examination), seizures, mental retardation (confirmed by psychometrics tests when available). Headaches were characterized as migraines or not-migraines according to the International Classification of Headache Disorders, 2nd edition. CSF data were collected when available (cells $>5 / \mathrm{mm} 3$, proteins $>0,4 \mathrm{~g} / \mathrm{dl}$, CSF opening pressure $>20 \mathrm{~mm} \mathrm{H}_{2} \mathrm{O}$ ). Brain MRI (T1, T2, T2 FLAIR, before and after injection of gadolinium, $\mathrm{T} 2 *$ weighted sequences, if available) at diagnostic and during followup, were read by two trained pediatric neurologist (NM and KD).

We reported the neurological outcome of these patients based on neurological examination, MRI results and audiometry tests during follow-up. We described their school performances: patients were considered to have learning difficulties if there were academic accommodations, grade repetition, early school leaving, or needing of special education classes. We also described the professional categories of the adult patients with CAPS based on the French National Institute of Statistics (INSEE) classification (Guide des nomenclatures professions et categories socio-professionnelles 2003).

We also analyzed the efficacy of canakinumab on neurological signs. The treatment was considered to be efficient if there was a complete resolution of headaches or if the frequency of these headaches was less than once a month. A partial response was defined if the headaches occured more than once a month. Hearing loss evolution under treatment was evaluated by repeated audiometry tests during follow-up: stable, improvement or worsening.

\section{Statistical analysis}

Statistical analysis was carried out with SPSS version 19.0. Parametric or nonparametric tests (Kruskal-Wallis and Mann-Whitney U tests) were used for continuous measurements as appropriate given normality. Differences were considered significant for $p$ values $<0.05$.

\section{Results}

Twenty-four patients (15 adults and 9 children at diagnosis) with CAPS were analyzed. The median age at disease onset was 0 year (birth) [range $0-14$ ], the median age at diagnosis was 20 years [range $0-53$ ] and the mean duration of follow-up was $10,4 \pm 2$ years. Two patients had no family history of CAPS.

Skin rash, musculo-skeletal involvement and fever were the most prevalent features as described in Table 1 and neurological involvement at diagnosis was noted in 17 patients $(71 \%)$. One patient had a mental retardation confirmed by neuropsychological tests (WISC IV), one patient had psychotic disorder. Fourteen (58\%) patients had learning difficulties needing academic accommodations (Table 1).

The $A 439 \mathrm{~V}$ mutation was frequently associated with no neurological signs and $R 260 \mathrm{~W}$ tended to be associated with neurological symptoms (Table 2).

All but one patient underwent at least one brain MRI before treatment. One brain MRI among 23 was abnormal before IL-1 $\beta$ treatment showing white matter lesions (Fig. 1a, b). The clinical examination of this 45-year-old female who had a heterozygous $R 260 \mathrm{~W}$ mutation revealed no clinical neurological abnormalities. These MRI lesions were characterized as multiple sclerosis-like lesions. Two lesions appeared on brain MRI after one year of treatment (Fig. 1c, d). After that, MRI remained stable as well as her clinical neurological examination and the patient was already reported in the literature by our group [10]. The MRI of one 25 year-old patient showed T2 lesions after seven years of canakinumab treatment and with no clinical consequences (Fig. 2). Lesions were compatible with a vasculitis. These 2 patients are from the same family and carry the same mutation $R 260 \mathrm{~W}$.

Five patients (21\%) underwent a CSF analysis; the opening pressure of the CSF was measured for 3 of them: 2 patients had an aseptic meningitis and the CSF opening pressure of one patient was elevated at $36 \mathrm{~mm}$ $\mathrm{H}_{2} \mathrm{O}$. These two patients had not migraine-headaches. 
Table 1 Characteristics of the patients with CAPS

\begin{tabular}{|c|c|c|c|}
\hline & & $n=24$ & $\%$ \\
\hline Female sex & & 14 & 58 \\
\hline Familial history of CAPS & & 22 & 92 \\
\hline \multirow[t]{4}{*}{ Genotype } & R260W & 12 & 50 \\
\hline & A439V & 4 & 17 \\
\hline & T348M & 4 & 17 \\
\hline & Other mutations & 4 & 17 \\
\hline Age at onset (years) & & $0[0-14]$ & \\
\hline Age at diagnosis (years) & & $20[0-53]$ & \\
\hline $\begin{array}{l}\text { Mean follow-up duration } \\
\text { (years) }\end{array}$ & & $10,4 \pm 1,94$ & \\
\hline Fever & & 22 & 92 \\
\hline \multirow[t]{3}{*}{ Skin involvement } & & 24 & 100 \\
\hline & Urticarial rash & 23 & 96 \\
\hline & Maculopapular rash & 17 & 71 \\
\hline Myalgia & & 15 & 62 \\
\hline Arthralgia & & 21 & 87 \\
\hline Conjunctivitis & & 20 & 83 \\
\hline \multirow[t]{7}{*}{ Neurological signs } & & 17 & 71 \\
\hline & Headaches & 15 & 62 \\
\hline & Migrainous headaches & 4 & 27 \\
\hline & $\begin{array}{l}\text { Chronic daily } \\
\text { headaches }\end{array}$ & 8 & 54 \\
\hline & Hearing loss & 10 & 59 \\
\hline & Optic nerve atrophy & 2 & 8 \\
\hline & Mental retardation & 1 & 0,04 \\
\hline Learning difficulties & & 14 & 58 \\
\hline Abnormal brain MRI $(n=23)$ & & 2 & 0,09 \\
\hline \multirow[t]{2}{*}{ CSF assays $(n=5)$} & Aseptic meningitis & 2 & 40 \\
\hline & $\begin{array}{l}\text { Elevated opening } \\
\text { pressure }\end{array}$ & 1 & 20 \\
\hline
\end{tabular}

The patients who had multiple sclerosis-like MRI lesions underwent after 8 months of imaging, a CSF analysis, which was normal.

Twenty-one patients (87\%) are treated with canakinumab (starting at a dose of $150 \mathrm{mg}$ every 8 weeks for adults and $2 \mathrm{mg} / \mathrm{kg}$ for children administrated as a subcutaneous injection). Treatment was not started in two children ( 9 and 4 years old) as their symptoms had very low

Table 2 Genotype correlation with neurological involvement

\begin{tabular}{lll}
\hline Genotype & $\begin{array}{l}\text { Neurological signs } \\
n=20\end{array}$ & $\begin{array}{l}\text { No neurological signs } \\
n=4\end{array}$ \\
\hline A439V (\%) & $1(25)$ & $3(75)$ \\
R260W (\%) & $12(60)$ & $0(0)$ \\
T348M (\%) & $3(15)$ & $1(25)$ \\
Other mutations (\%) & $4(20)$ & $0(0)$ \\
\hline
\end{tabular}

impact in their everyday life. One 42 year-old female patient still refuses the treatment.

Fourteen (82\%) of the treated patient described improvement of their headaches: a complete resolution of headaches in 12 patients $(80 \%)$ and partial resolution in $2(13 \%)$ was observed. One patient had no improvement of his migrainous headaches under treatment. The elevated opening CSF pressure of one patient, normalized two years after treatment onset.

Eight $(80 \%)$ patients reported improvement $(n=3)$ or stabilization $(n=5)$ of their hearing loss proven by audiometry during follow up.

At follow-up, 6 patients were less than 18 years old, among them 3 (50\%) have learning difficulties needing school accommodations, and $3(50 \%)$ have no school difficulties. The professional categories of adult patients $(\mathrm{n}=18)$ are described in (Fig. 3). Eleven adult patients (61\%) underwent school difficulties, at follow-up 3 have no jobs, 3 are workers, 1 is a skilled worker and 4 are employees. Among adults who had no school difficulties, 4 are post-graduate students, 1 is an employee and 2 are skilled workers (Fig. 3).

\section{Discussion}

In this retrospective observational study, neurological involvement concerns $71 \%$ of patients with CAPS, mostly with headaches and neurosensorial hearing loss. Canakinumab treatment confirms its efficacy in neurological symptoms in $76 \%$ of treated patients [4]. The most important findings in our study is the impact of CAPS on school performances: $50 \%$ of patients under 18 years old with CAPS have learning difficulties needing school accommodations, and $61 \%$ of adult patients had school difficulties with probable consequences in their professional careers.

In the largest CAPS study based on Eurofever registry $(n=136)$, neurological manifestations were observed in $40 \%$ and is mostly represented by headaches (70\%), papilledema (52\%), meningitis (26\%), hydrocephalus (18\%), mental retardation (16\%), seizures (4\%) [9]. Hearing loss which is secondary to a cochlear inflammation represented $42 \%$ and was not included in the neurological symptoms, which can explain the underestimation of neurological involvement [9]. In our study, we confirmed that headaches and hearing loss were the most prominent neurological symptoms,

The mechanism of headaches in patients with CAPS is not well known. In our study, 4 patients (27\%) had migrainous headaches and 2 patients with non-migrainous headaches had an aseptic meningitis and one of them had a high opening CSF pressure showing an intracranial hypertension which can explain the headaches [11]. Only $21 \%$ of our patients underwent CSF analysis and CSF opening pressure was measured only for 2 patients. 

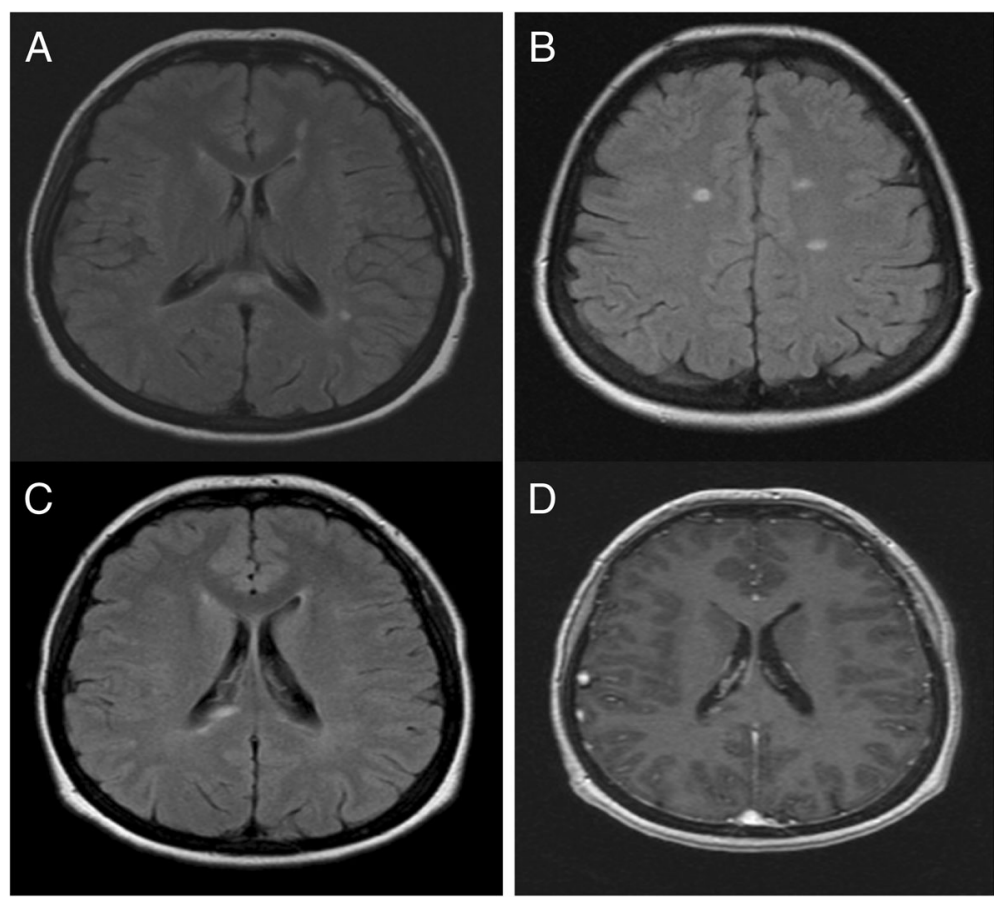

Fig. 1 Brain MRI lesions of 52 year-old patient with CAPS before and after treatment. Axial FLAIR brain MRI images showing well limited periventricular (a), corpus callosum (a) and juxtacortical lesions (b). Brain MRI images with Axial FLAIR sequences (c) and T1 after gadolinium injection sequences (d), after one year of canakinumab treatment, of the same patient showing new FLAIR right paraventricular abnormalities with Gadolinium enhancement

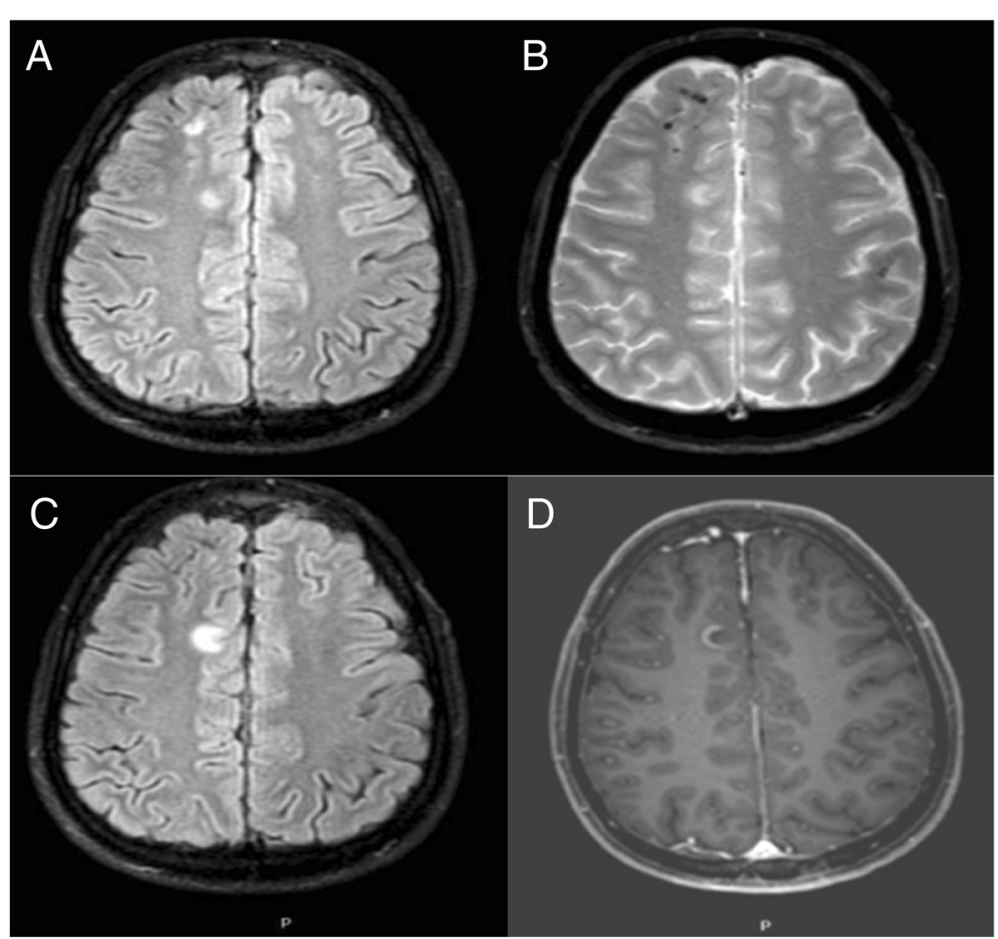

Fig. 2 MRI images of 26 year-old patient with CAPS during a relapse with severe headaches. (a): Axial Flair images showing T2 FLAIR hypersignal right frontal juxta-cortical lesions with (b) hyposignal lesions on T2* weighted sequences suggesting hemosiderin deposits and (c) right anterior cingular gyrus T2 FLAIR hypersignal with (d) gadolinium enhancement 


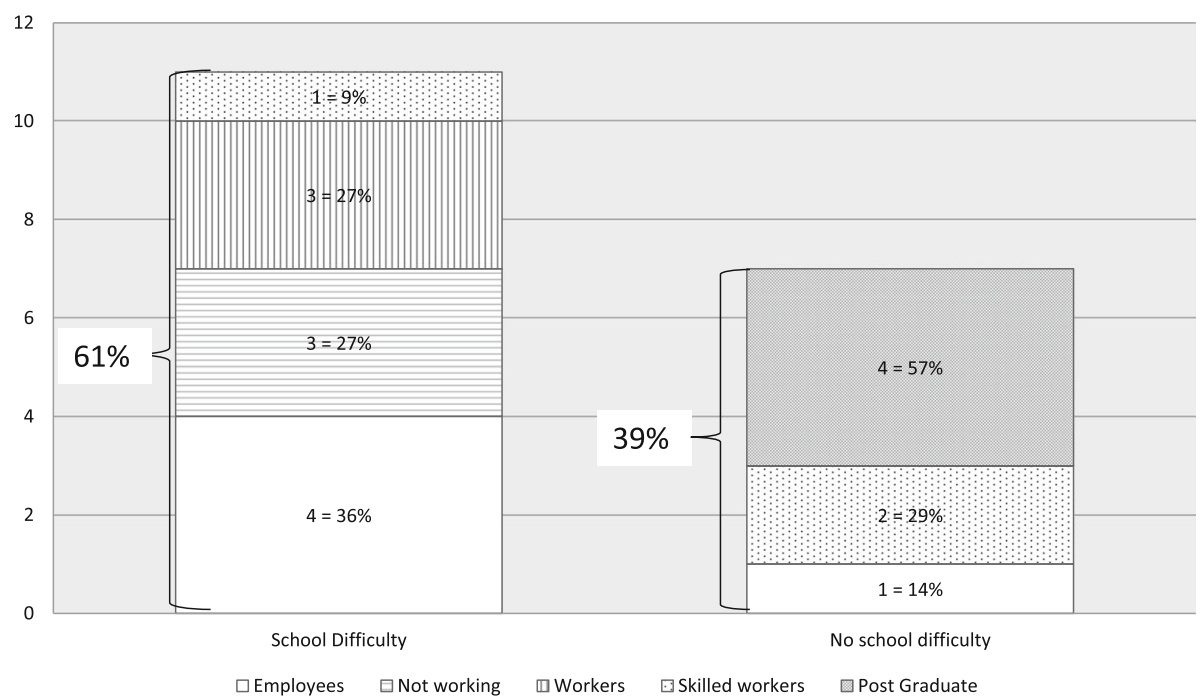

Fig. 3 Professional categories of adults with CAPS $(n=18)$

Unfortunately, CSF assays was not exhaustive and intracranial hypertension and or chronic meningitis might be under-estimated. A high level of pro-inflammatory cytokines including IL-1 $\beta$ in patients with migraines had been described [12]. This may be relevant to understand the physiopathological mechanism of headaches in CAPS and can explain canakinumab efficacy which was complete for 2 of 4 patients with migrainous headaches.

Fifty nine percent of the patients with CAPS have a hearing loss. The physiopathology is not well known, it has been observed that some children with CINCA had a cochlear inflammation demonstrated on MRI, after IL-1 receptor antagonist treatment there was an improvement of cochlear enhancement [6]. In our study, no cochlear inflammation has been described on MRI but this imaging was not specifically performed for inner ear disease, and cochlear abnormality on MRI may have not been seen.

Before any anti- IL-1 $\beta$ treatment, one patient had white matter lesions with demyelination as it may be seen in multiple sclerosis without any neurological sign (except for chronic headaches) unlike the only other CAPS patient described in literature with demyelinating disease who had a hemiparesia [13]. In MS, it has been suggested that brain lesions may also be induced following inflammatory synaptopathy and neurodegeneration caused by IL-1 $\beta$ [14] and a similar mechanism may explain brain lesions in patients with CAPS syndrome. Recent studies suggested that IL-1 receptor antagonist (IL-1Ra) could cross the blood-brain barrier and have a neuroprotective effects in rodent model as well as in patients with ischemic stroke or subarachnoid hemorrhage brain hemorrhage [15]. Under anti-IL-1 $\beta$, in one patient with white matter lesions before the start of treatment, 2 new lesions appeared after one year of treatment. Thereafter, all brain MRI of this patient remained stable suggesting that the lesion may eventually be associated with the disease and treatment may have an effect on long term.

Although we could not perform statistical test du to small numbers of included patients, we have observed that $A 439 \mathrm{~V}$ mutation is frequently associated with a non-neurological phenotype as previously published [9]. Interestingly, we also noticed that $R 260 \mathrm{~W}$ mutation, tend to be associated with neurological involvement. Moreover 2 members of the same family with this mutation had brain MRI lesions suggesting that brain lesions in these patients may be linked to the disease. Larger studies are needed to detect any specific pattern of these lesions.

Our study described the neurological outcome under anti- IL-1 $\beta$ treatment with a mean duration of follow-up of 10,4 years. Canakinumab had shown its sustained efficacy on neurological symptoms: $82 \%$ improved their headaches under treatment, and $80 \%$ improved or stabilized their hearing loss. In our study the median age at onset is birth and the median age at diagnosis is 20 years. There is an important delay of diagnosis and therefore a delay for treatment, already noted in previous studies [9]. This highlights the importance of recognizing the clinical features of this rare syndrome for neonatologists, pediatrician and pediatric neurologist in order to initiate the specific treatment and reverse systemic symptoms as well as neurological symptoms and prevent progression of them, especially headaches and hearing loss.

Our study focused on school performances and professional career during follow-up. Although there is only one objective mental retardation, school difficulties are 
frequent in children with CAPS. The cognitive difficulties may be under-estimated: only 4 patients underwent psychometric tests. The impact of CAPS in school and professional performances is multifactorial: chronic pains, school absences, sick leaves are also implicated. A recent study showed on 7 patients in a prospective monocentric study that canakinumab had not only a sustained effect on quality of life, but also allowed dramatic changes in social and work lives [16]. The benefit of canakinumab treatment in allowing a professional life was one of their most important observations; no patients were out of work during the follow-up (4.8 \pm 0.8 years). The gain in vitality scores permitted them to enjoy a social life after work and physical activity, which were impossible without treatment. Psychometric tests, neuropsychological evaluation, neuropediatric follow-up is not systematic for children with CAPS, although the learning difficulties are frequent in these patients as shown in our study.

\section{Conclusion}

We provided a detailed description of the neurological involvement, which is frequent in adults and children with CAPS. Canakinumab seems to have changed the general outcome of these patients as well as the neurological outcome. Further studies in larger cohorts of children with CAPS focusing in intellectual efficiency and school performances are necessary. Because of the frequency of school difficulties shown in our study in children with CAPS, the role of the pediatric neurologist in the muldisciplinary follow-up of these patients is very important and regular neuropsychological evaluations and neurological following are advisable for children with CAPS.

\section{Abbreviations \\ CAPS: Cryopyrin-associated periodic syndrome; CINCA: Chronic Infantile Neurologic Cutaneous Articular syndrome; CSF: Cerebro-spinal fluid; MRI: Magnetic resonance imaging; MWS: Muckle-Wells syndrome; NOMID: Neonatal Onset Multisystem Inflammatory Disease}

\section{Acknowledgements}

Not applicable.

\section{Funding}

No funding was obtained for this research.

\section{Availability of data and materials}

The datasets during and/or analysed during the current study available from the corresponding author on reasonable request.

\section{Authors' contributions}

NM acquisition of data, analysis and interpretation of data, drafting the article. HM and IM acquisition of data, revising the article critically. IKP and KD substantial contributions to conception and design, acquisition of data, analysis and interpretation of data, drafting the article and revising it critically. All authors read and approved the final manuscript.
Consent for publication

Patients have given their consent to electronic storage and treatment of their medical data.

Ethics Approval and Consent to Participate

According to our national regulations, no IRB approval was necessary.

\section{Financial disclosures}

NM, HM, IM have no financial disclosures. IKP has received consulting fees from Novartis, Abbvie, Pfizer and Sobi. KD has received consulting fees from Novartis.

\section{Author details}

${ }^{1}$ Department of Pediatrics, Meaux General Hospital, 77104 Meaux Cedex, France. ${ }^{2}$ Assistance Publique-Hôpitaux de Paris, Hôpitaux Universitaires Paris Sud, Department of Pediatrics, Pediatric Rheumatology, National Referral Centre of Auto-inflammatory Diseases, CEREMAl, CHU Bicêtre, University of Paris Sud, Le Kremlin Bicêtre, France. ${ }^{3}$ Assistance Publique-Hôpitaux de Paris, Hôpitaux Universitaires Paris Sud, Pediatric Neurology Department and National Referral Center for Neuroinflammatory Diseases in Children and Inserm UMR 1184, Center for immunology of viral infections and autoimmune diseases, CEA, IDMIT, University Paris Sud, 63, rue Gabriel Péri, 94276 Le Kremlin-Bicêtre Cedex, France.

Received: 19 September 2016 Accepted: 7 February 2017

Published online: 14 February 2017

\section{References}

1. Cuisset L, Jeru I, Dumont B, Fabre A, Cochet E, Le Bozec J, Delpech M, Amselem S, Touitou I. French Csg: Mutations in the autoinflammatory cryopyrin-associated periodic syndrome gene: epidemiological study and lessons from eight years of genetic analysis in France. Ann Rheum Dis. 2011;70(3):495-9.

2. Hoffman HM, Mueller JL, Broide DH, Wanderer AA, Kolodner RD. Mutation of a new gene encoding a putative pyrin-like protein causes familial cold autoinflammatory syndrome and Muckle-Wells syndrome. Nat Genet. 2001;29(3):301-5.

3. Jesus AA, Goldbach-Mansky R. IL-1 blockade in autoinflammatory syndromes. Annu Rev Med. 2014;65:223-44.

4. Lachmann HJ, Kone-Paut I, Kuemmerle-Deschner JB, Leslie KS, Hachulla E, Quartier P, Gitton X, Widmer A, Patel N, Hawkins PN, et al. Use of canakinumab in the cryopyrin-associated periodic syndrome. N Engl J Med. 2009;360(23):2416-25.

5. Muckle TJ. Wellsm: Urticaria, deafness, and amyloidosis: a new heredofamilial syndrome. Q J Med. 1962;31:235-48.

6. Goldbach-Mansky R, Dailey NJ, Canna SW, Gelabert A, Jones J, Rubin BI, Kim HJ, Brewer C, Zalewski C, Wiggs E, et al. Neonatal-onset multisystem inflammatory disease responsive to interleukin-1 beta inhibition. N Engl J Med. 2006;355(6):581-92.

7. Hoffman HM, Wanderer AA, Broide DH. Familial cold autoinflammatory syndrome: phenotype and genotype of an autosomal dominant periodic fever. J Allergy Clin Immunol. 2001;108(4):615-20.

8. Kone-Paut I. Cryopyrine-associated periodic syndrome: CAPS seen from adulthood. Rev Med Interne. 2015;36(4):277-82.

9. Levy R, Gerard L, Kuemmerle-Deschner J, Lachmann HJ, Kone-Paut I, Cantarini L, Woo P, Naselli A, Bader-Meunier B, Insalaco A, et al. Phenotypic and genotypic characteristics of cryopyrin-associated periodic syndrome: a series of 136 patients from the Eurofever Registry. Ann Rheum Dis. 2015;74(11):2043-9.

10. Compeyrot-Lacassagne S, Tran TA, Guillaume-Czitrom S, Marie I, Kone-Paut I. Brain multiple sclerosis-like lesions in a patient with Muckle-Wells syndrome. Rheumatology (Oxford). 2009:48(12):1618-9.

11. Kitley JL, Lachmann HJ, Pinto A, Ginsberg L. Neurologic manifestations of the cryopyrin-associated periodic syndrome. Neurology. 2010;74(16):1267-70

12. Perini F, D'Andrea G, Galloni E, Pignatelli F, Billo G, Alba S, Bussone G, Toso $\checkmark$. Plasma cytokine levels in migraineurs and controls. Headache. 2005:45(7):926-31.

13. Lequerre $T$, Vittecoq $O$, Saugier-Veber $P$, Goldenberg $A$, Patoz $P$, Frebourg $T$, Le Loet X. A cryopyrin-associated periodic syndrome with joint destruction. Rheumatology (Oxford). 2007:46(4):709-14.

14. Rossi S, Motta C, Studer V, Macchiarulo G, Volpe E, Barbieri F, Ruocco G, Buttari F, Finardi A, Mancino R, et al. Interleukin-1 beta causes excitotoxic 
neurodegeneration and multiple sclerosis disease progression by activating the apoptotic protein p53. Mol Neurodegener. 2014;9:56.

15. Singh N, Hopkins SJ, Hulme S, Galea JP, Hoadley M, Vail A, Hutchinson PJ, Grainger S, Rothwell NJ, King AT, et al. The effect of intravenous interleukin1 receptor antagonist on inflammatory mediators in cerebrospinal fluid after subarachnoid haemorrhage: a phase II randomised controlled trial. J Neuroinflammation. 2014;11:1

16. Marsaud C, Marie I, Kone-Paut I. Longterm followup of quality of life in patients with cryopyrin-associated periodic syndrome treated with canakinumab, an anti-interleukin 1 beta monoclonal antibody. J Rheumatol. 2014:41(8):1721-2

Submit your next manuscript to BioMed Central and we will help you at every step:

- We accept pre-submission inquiries

- Our selector tool helps you to find the most relevant journal

- We provide round the clock customer support

- Convenient online submission

- Thorough peer review

- Inclusion in PubMed and all major indexing services

- Maximum visibility for your research

Submit your manuscript at www.biomedcentral.com/submit
) Biomed Central 